\title{
Efficient Fluorescence Quenching by Distant Production of a Free Electron
}

Andreas Hans, ${ }^{* \dagger}{ }^{\dagger}$ Philipp Schmidt, ${ }^{\dagger}$ Christian Ozga, ${ }^{\dagger}$ Clemens Richter,,${ }^{\ddagger}$ Huda Otto, ${ }^{\dagger}$ Xaver Holzapfel, ${ }^{\dagger}$ Gregor Hartmann, ${ }^{\dagger}$ Arno Ehresmann, ${ }^{\dagger}$ Uwe Hergenhahn, ${ }^{\ddagger}$, and André Knie ${ }^{*, \dagger}$

$\dagger$ University of Kassel, Institute of Physics and Center for Interdisciplinary Nanostructure

Science and Technology (CINSaT), Heinrich-Plett-Str. 40, 34132, Kassel, Germany

$\ddagger$ Leibniz Institute of Surface Engineering (IOM), Permoserstr. 15, 04318 Leipzig, Germany

IDepartment of Physics, Free University Berlin, Arnimallee 14, 14195 Berlin, Germany

$\S$ Max Planck Institute for Plasma Physics, Wendelsteinstr. 1, 17491 Greifswald, Germany

E-mail: hans@physik.uni-kassel.de; knie@physik.uni-kassel.de 


\begin{abstract}
Energy and charge transfer processes play an important role in many fundamental reactions in chemistry, biochemistry, and even technology. If an entity being part of a larger system is photoexcited, its energy will dissipate, for example by rearrangement of electron density in a large molecule, or by photon emission (fluorescence). Here, we report about the experimental observation of free electrons from a heterogeneous van der Waals cluster, in which some sites act as electron emitters receiving their energy efficiently from other 'antenna' sites that are resonantly excited in the UV range. By complementing electron spectroscopy with fluorescence detection, we can directly observe that electron emission via this mechanism completely quenches fluorescence once the channel opens. We suggest this mechanism to be important for both quenching of fluorescence as well as resonantly enhancing free electron production in a variety of systems.
\end{abstract}

\title{
Graphical TOC Entry
}

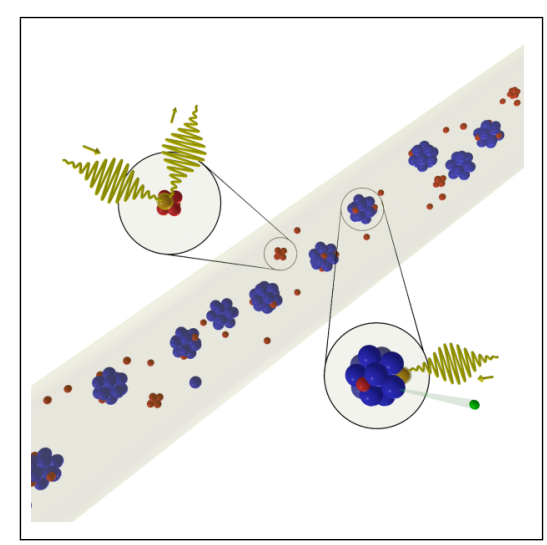


Ever since the discovery of the photoelectric effect, ${ }^{1}$ i.e. the release of a free electron initiated by absorption of a photon, the efficiency of this mechanism has been subject of intense research. ${ }^{2-4}$ Photoionization is of fundamental importance in many fields of physics, chemistry, and biochemistry and nowadays, many complex technological applications rely on this mechanism.

Direct photoionization in the single-photon interaction regime is a non-resonant process, where the only condition the ionizing radiation of frequency $\nu$ has to fulfill is that the energy $E=h \nu$ of the single photon has to be larger than the ionization energy $E_{I}$ of the respective material. This results in a step-like photoionization cross section at the energetically lowest ionization threshold and a relatively smooth behavior with increasing photon energy. For higher thresholds, also autoionizing resonances in the ionization continuum are common. ${ }^{5,6}$ Resonant excitation of valence electrons below the ionization threshold typically leads to fluorescence emission, in molecules possibly accompanied by nuclear rearrangement and/or dissociation. If a system (e.g. atom or molecule) is embedded in an environment, several non-local ionization processes have to be considered when discussing the ionization pathways. In weakly bound matter, like van der Waals or hydrogen bound clusters or liquids, such mechanisms were recently investigated intensely. ${ }^{7,8}$ In processes like interatomic Coulombic decay (ICD) or electron-transfer mediated decay (ETMD), ionization typically occurs if a system is excited into states which energetically lie above its ionization energy, but below the one-site double ionization potential. Since the two-site double ionization potential often is considerably lower, further ionization can be achieved by transferring energy between sites. ${ }^{9}$

Having such mechanisms in mind, it is straightforward that ICD in an extended system can occur, if the energy stored on a site A is above the ionization energy of a partner B, although it is below the ionization energy of A. The cross section of photoionization of site B can thereby be enhanced tremendously, possibly by orders of magnitude, if site A is resonantly excited by incoming radiation. Site A acts as an antenna to receive energy and then transfers it to its neighbor. 


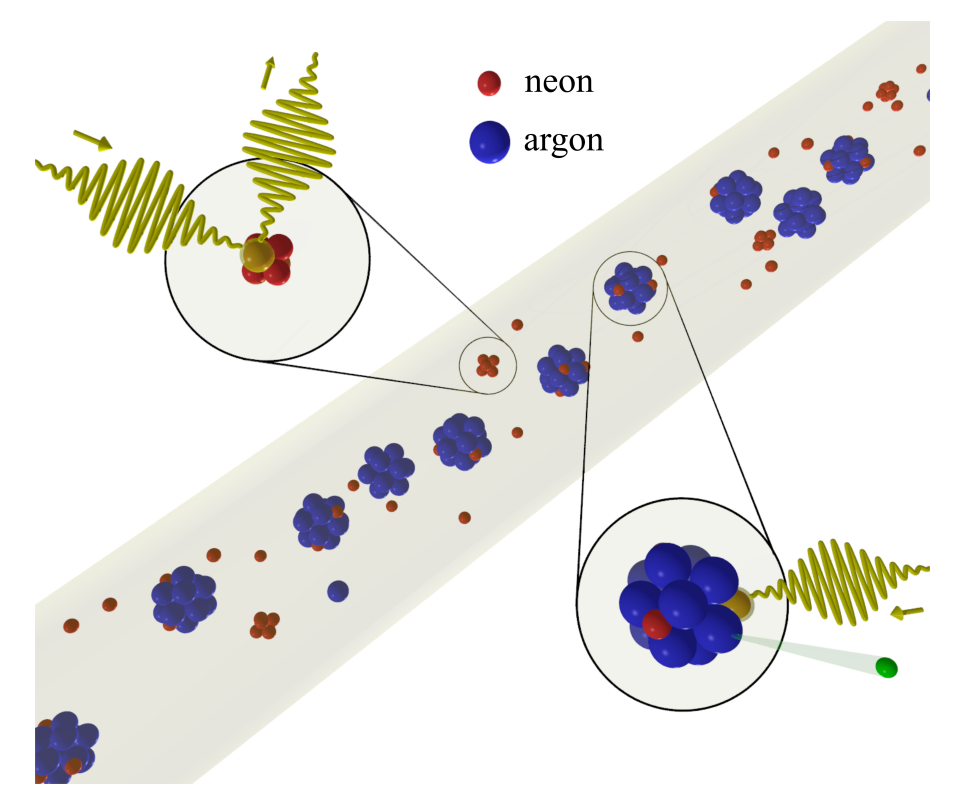

Figure 1: Sketch of the process under investigation. In a pure Ne cluster, resonant outer valence excitation is followed by fluorescence emission (upper left magnification). Contrary, in heterogeneous Ne-Ar clusters, the energy is transferred to ionize a neighbouring Ar atom (lower right magnification), leading to a resonantly enhanced ionization cross section of Ar.

Such processes have been observed by detection of ionic fragments in pioneering works targeting small noble gas-benzonitrile clusters ${ }^{10}$ Ar-water clusters, ${ }^{11}$ doped He droplets ${ }^{12}$ and He-Ne dimers. ${ }^{13,14}$ We consider the energy transfer mechanism to be a regular member of the variety of ICD processes, but note that this process has been termed also "two-center resonant photoionization" 15 or "excitation transfer ionization" 16 in some contexts. Note that this process is considerably different to the more commonly discussed Penning ionization, in which ionization is usually mediated in collisions of excited atoms with neutrals. ${ }^{17}$ In this article, we report the direct observation of the ICD-like energy transfer in large Ne-Ar clusters. The process is sketched in Figure 1. We directly measure the resonantly enhanced photoemission cross section of Ar, and the accompanying quenching of fluorescence from excited Ne.

Heterogeneous Ne-Ar clusters were produced by supersonic co-expansion of Ne gas admixed with a controlled amount of Ar atoms (see experimental section). The development of the outer valence resonances in Ne clusters, corresponding to the atomic $2 \mathrm{p}_{1 / 2,3 / 2} \rightarrow 3 \mathrm{~s}$ 


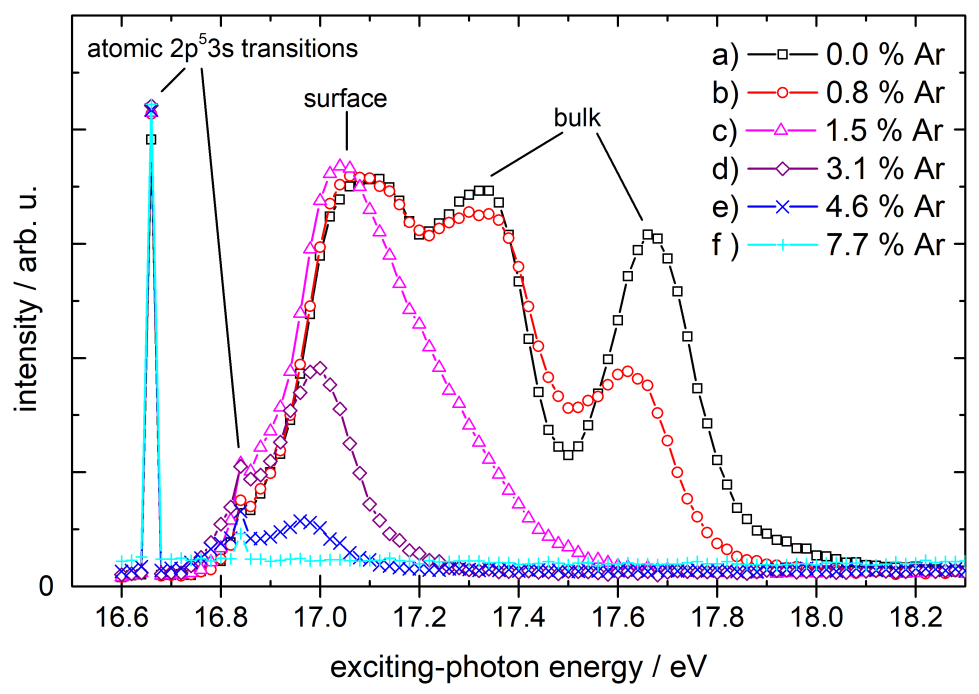

Figure 2: VUV fluorescence yield as function of the exciting-photon energy for different mixing ratios of a co-expanding Ne-Ar gas mixture. The Ar content (volume percentage) in the expanding gas mixture is given in the inset, resulting in differently composited (heterogeneous) clusters. Features appearing due to excitation of atomic Ne or surface and bulk components of Ne clusters are labelled. ${ }^{18}$

transitions (at about $16.67 \mathrm{eV}$ and $16.84 \mathrm{eV}$ ), is known and has been investigated by fluorescence spectroscopy. ${ }^{18}$ These resonances are energetically below the Ne $2 p$ ionization energy, and therefore decay by fluorescence. The resonances of clusters are blue-shifted with respect to the atomic resonances, with three distinct main components in the range from $16.8 \mathrm{eV}$ to $18.0 \mathrm{eV}$. Since the components can be attributed to excitation of bulk or surface sites of the cluster ${ }^{18}$ their relative intensity is an indicator for the mean size of the clusters in the beam under investigation.

The fluorescence excitation function of the pure Ne cluster jet in the present experiment is shown in Figure 2a. Comparing to literature spectra, the signal corresponds to a jet with a size distribution around a medium mean cluster size. ${ }^{18}$ The exact size of each cluster does not affect the interpretation presented here. As apparent from Figures 2b-f, significant changes appear in the excitation function, if the Ar admixture is enhanced stepwise from $0 \%$ to $7.7 \%$. Note that the given mixing ratios present the composition of the co-expanding gas mixture, not the final composition of the clusters. ${ }^{19,20}$ For low Ar admixture, the bulk 
component decreases drastically and already vanishes for a ratio of $98.5 \% \mathrm{Ne}$ and $1.5 \% \mathrm{Ar}$ (Figure 2c). If the amount of $\mathrm{Ar}$ is increased further, the intensity of the surface component also decreases until only the $2 \mathrm{p} \rightarrow 3$ s fine structure components of atomic Ne remain, at 7.7\% Ar admixture in the gas reservoir. The overall expansion conditions, i.e. stagnation pressure and nozzle temperature, were not changed during this series of excitation functions. The efficient fluorescence quenching therefore cannot be caused by a lack of condensed Ne. Instead, with higher Ar admixture, the probability for incorporation of Ar atoms into a cluster rises, in particular because for the given expansion conditions the condensation of $\mathrm{Ar}$ is much more likely than Ne condensation. ${ }^{19}$ We conclude that upon increase of the $\mathrm{Ar}$ fraction, only some remaining small clusters of pure Ne fluoresce, because all larger clusters contain one or several Ar atoms and decay by the proposed ICD mechanism.

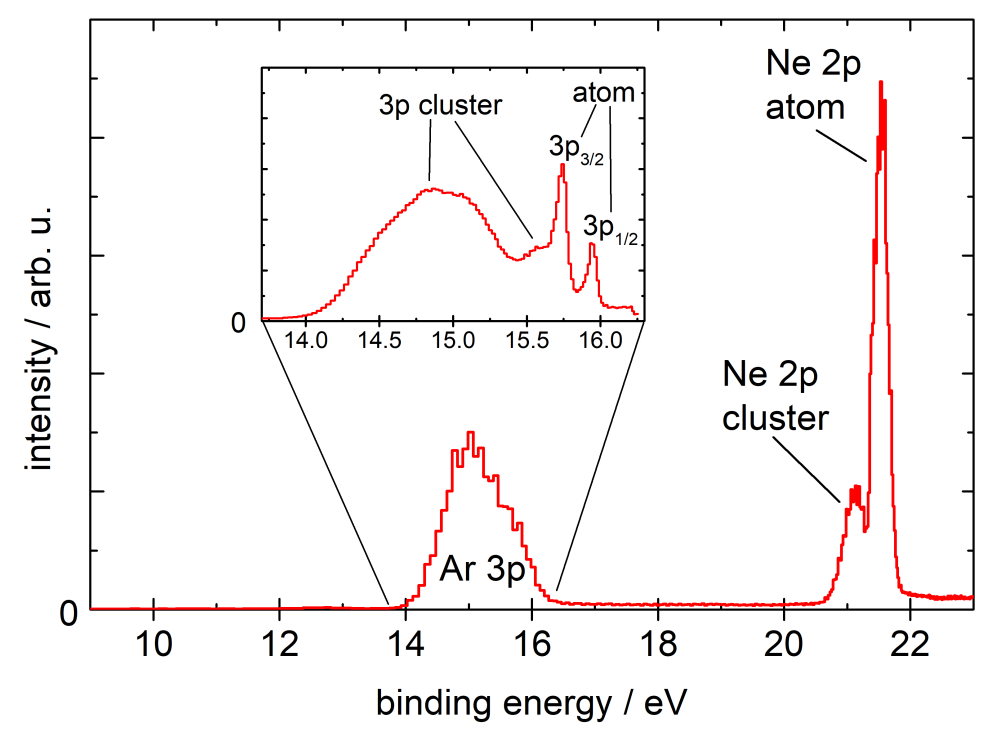

Figure 3: Electron spectrum of a heterogeneous Ne-Ar cluster jet produced by a co-expanding gas mixture containing $5 \%$ Ar, using 0.58 bar stagnation pressure and $65 \mathrm{~K}$ nozzle temperature and recorded at $23 \mathrm{eV}$ photon energy. The contribution from Ar atoms and clusters cannot be separated in the overall valence electron spectrum. Therefore, another electron spectrum was recorded at identical expansion parameters but with a low retardation voltage, repelling the Ne electrons and increasing the resolution in the range of Ar 3p binding energies. The inset shows the spectrum of Ar electrons, resolved for contributions from clusters and atoms, including the fine structure.

To characterize the cluster jet composition and unambiguously identify the fluorescence 
quenching mechanism, we have performed a second experiment in which the electron spectra of a comparable cluster jet were measured. The valence electron spectrum of a cluster jet produced by co-expansion of $95 \% \mathrm{Ne}$ and $5 \% \mathrm{Ar}$, ionized by photons of $23 \mathrm{eV}$ energy, is shown in Figure 3. The spectrum consists of three distinct features: the $2 \mathrm{p}$ photoelectron line of atomic Ne at $21.6 \mathrm{eV}$ binding energy, a small amount of 2p photoelectrons from condensed $\mathrm{Ne}$ with maximum at about $21.0 \mathrm{eV}$, and electrons from Ar with a maximum at about $15.0 \mathrm{eV}$. The valence spectra of Ne-Ar clusters have earlier been used to investigate the geometric structure of such clusters. ${ }^{19}$ Electrons from Ar clusters and atoms cannot be resolved with the spectrometer settings used to obtain the full valence spectrum of Figure 3, but a retarding voltage was used to zoom into the Ar electrons for the same cluster jet conditions. The separated peaks from atoms and clusters as well as the $3 \mathrm{p}_{1 / 2,3 / 2}$ fine structure splitting are visible in the inset of Figure 3. By comparing the areas of atomic and cluster contributions it can be estimated, that about $90 \%$ of the Ar is condensed in clusters. Similar electron spectra of pure Ar clusters and mixed Ne-Ar clusters have been recorded earlier. ${ }^{19,20}$

In Figure 4a, the photoelectron spectrum of the heterogeneous clusters for a series of photon energies is shown. With the settings of the electron spectrometer used for this measurement, atomic and cluster contribution cannot be separated, however, because of the high condensation (see Figure 3) the Ar feature can be regarded as originating basically from Ar clusters. The onset of the electrons from Ar clusters seems to be somewhat shifted to higher binding energies compared to the spectrum of Figure 3. As the target parameters were identical in both measurements, this is most likely an artefact of the reduced energy resolution for higher kinetic energies of the electrons, since the spectrum in Figure 3 was recorded at $4.5 \mathrm{eV}$ to $6.5 \mathrm{eV}$ higher photon energies than the spectra of Figure 4.

The total emission cross section of electron from Ar in Figure 4b shows overall little structure, exhibiting only one broad maximum at about $17 \mathrm{eV}$. Position and shape of this maximum match well with the fluorescence excitation function of small Ne clusters, which is also shown for comparison. However, the maximum in the electron yield is caused by 
resonant excitation of a Ne atom sitting on the surface of a large Ar cluster. As the Ne assembles as a shell around the Ar core,${ }^{19}$ it is reasonable to expect a signal shape which is similar to that of small Ne clusters.

The photoelectron spectrum of pure Ar clusters has also been investigated in detail in the excitation energy range between $16.8 \mathrm{eV}$ and $18.0 \mathrm{eV},{ }^{21}$ coinciding with the Ne valence excitations which are of interest in this work. An energy dependent structure of the 3p photoelectron line was reported and assigned to an energy dispersion of the valence band. Although weak changes in the spectrum off resonance can be found in the present experiment, this feature is not observed with a comparable prominence. This might suggest that the energy band dispersion is a surface effect. However, the present discussion of the signal from the heterogeneous clusters is not affected by that.

Regarding the efficiency of the ionization process by energy transfer from resonantly excited Ne, we suppose it to be orders of magnitude faster than the competing radiative decay in pure Ne. The radiative lifetime of the $2 \mathrm{p}^{5} 3$ s state in atomic Ne is about $1.6 \mathrm{~ns},{ }^{22}$ while the electron emission happens on a femtosecond to picosecond time scale. From the relatively high abundance of Ar compared to condensed Ne in the electron spectrum (Figure 3), we can assume that all Ne clusters contain bound Ar also, which can serve as partner for the energy transfer. It has been investigated before, that ICD-like processes are possible also with non-nearest neighbors. ${ }^{23,24}$ The energy transfer leading to ionization can therefore be regarded as an ultra-efficient quenching mechanism in loosely bound matter. Because the Ne resonance is energetically above the Ar ionization threshold, the ionization mediated by energy transfer can interfere with direct Ar photoionization. ${ }^{5,15}$ Indeed, an asymmetric line shape is observed in Figure 3b. However, the influence of cluster size and composition distributions and interference effects cannot be disentangled from the present data.

As can be deduced from Figure 4b, the ionization cross section of the co-expanded NeAr jet is enhanced by a factor of about 3 on top of the Ne resonance, compared to the direct photoionization of Ar. We therefore envision this process also to be of relevance for 


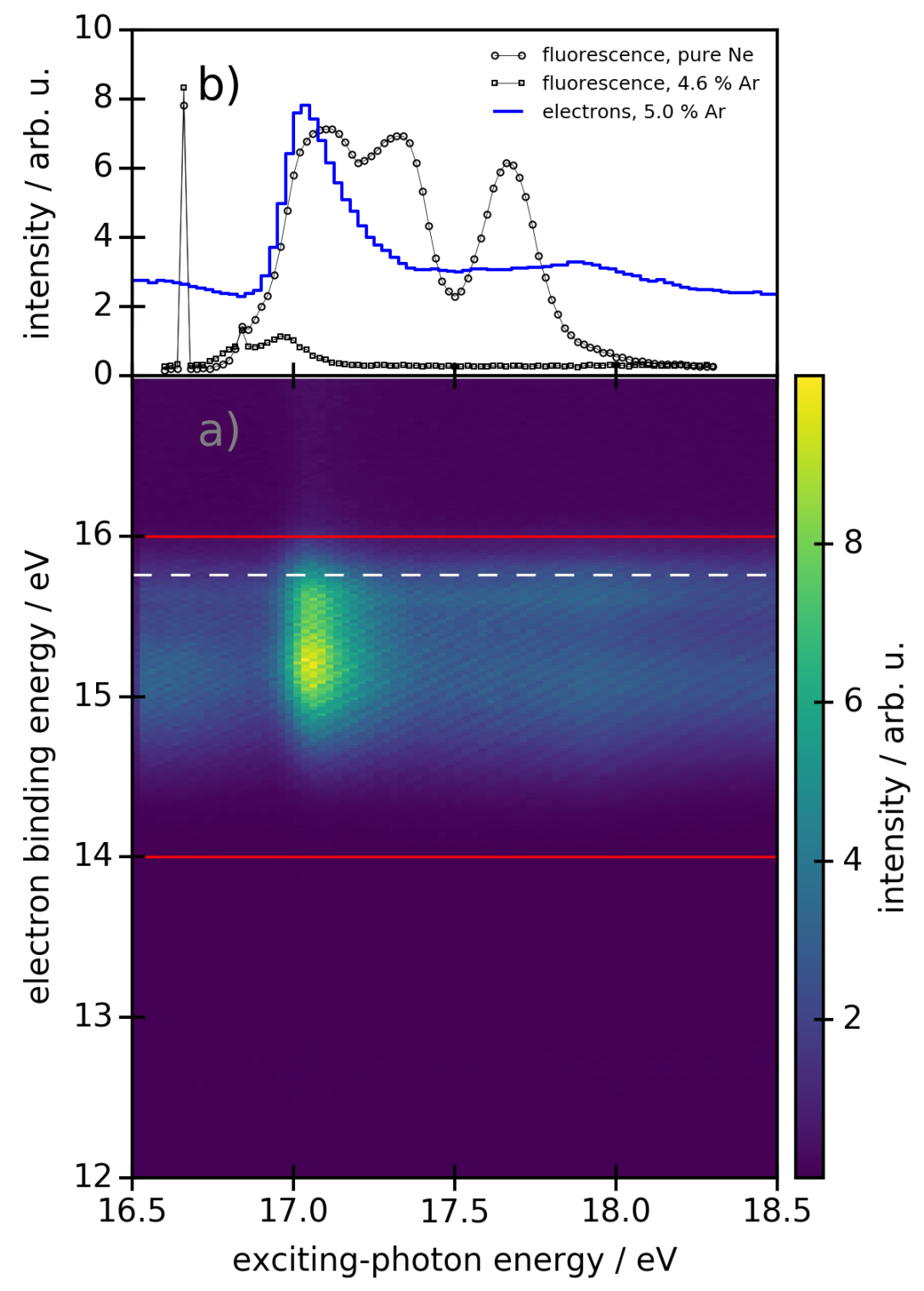

Figure 4: a) Color-coded electron spectrum from heterogeneous Ne-Ar clusters for one set of expansion parameters (see text) as function of the exciting-photon energy in the range of $\mathrm{Ne}$ cluster valence excitations. The dashed white line indicates the atomic $\operatorname{Ar} 3 \mathrm{p}_{3 / 2}$ ionization threshold. b) Integrated yield of electrons (blue solid line) with binding energies between $14 \mathrm{eV}$ and $16 \mathrm{eV}$ (area between red solid lines in panel a). For comparison, the fluorescence yield from Figure 2 is also shown for pure Ne (open circles) and $4.6 \%$ Ar admixture (open squares). 
artificially enhancing the ionization cross section of a given material by dopant substances which resonantly absorb the radiation. As the increase in ionization cross section is caused by resonant excitation, the mechanism could be exploited to tailor systems with unique, photon energy dependent ionization characteristics in both fundamental research and technology.

In conclusion, we directly observed the resonantly enhanced production of free electrons by ICD from excited states of one species into the ionization continuum of a van der Waals bound partner. Any radiative decay from the excited states is quenched by this process. The mechanism might be the origin of many fluorescence quenching processes and could perspectively be exploited for tailored ionization cross sections in a variety of weakly bound systems.

\section{Experimental Methods}

The experiments were performed at the UE112 PGM-1 beamline of BESSY II, HelmholtzZentrum Berlin (HZB). Two different setups were used for measuring the resonance-enhanced photoionization and the fluorescence quenching. Both setups have been discussed earlier in great detail and will only briefly be described here. For electron detection, a magnetic bottle type time-of-flight spectrometer was used. ${ }^{25}$ A supersonic jet of co-expanding Ne and Ar gas with initial mixing ratio of $95 \% \mathrm{Ne}$ and $5 \%$ Ar was used, resulting in a stagnation pressure of 0.58 bar behind the conical copper nozzle of $80 \mu \mathrm{m}$ diameter. The nozzle was cooled to $65 \pm 1 \mathrm{~K}$ using a liquid He cryostat. After passing through a $1 \mathrm{~mm}$ skimmer, the cluster jet was crossed with circularly polarized synchrotron radiation. The storage ring was operated in single bunch mode, providing 800 ns between bunches to measure the time-of-flight spectra. To record the yield of Ar electrons as a function of the exciting-photon energy, the latter was scanned stepwise and a full electron spectrum was recorded for each energy step. The fluorescence quenching was observed using a similar setup and an open-face microchannel

plate detector sensitive for vacuum-ultraviolet radiation as described recently. ${ }^{26}$ Here, the 
clusters were produced by co-expansion of a Ne-Ar mixture with reservoir pressure 4.5 bar through a conical nozzle of $40 \mu \mathrm{m}$ diameter, which was cooled to $120 \pm 1 \mathrm{~K}$ using liquid $\mathrm{N}_{2}$. The synchrotron was operated in multibunch mode, the exciting-photon energy scanned stepwise and the yield of fluorescence recorded for each step.

\section{Acknowledgement}

We gratefully acknowledge beamtime allocation by HZB and support of the BESSY II staff during the experiments. This work was supported by the Deutsche Forschungsgemeinschaft through Research Unit FOR 1789 and Sonderforschungsbereich 1319.

\section{References}

(1) Einstein, A. Über einen die Erzeugung und Verwandlung des Lichtes Betreffenden Heuristischen Gesichtspunkt. Ann. Phys. 1905, 322, 132-148.

(2) Burke, P. G.; Kleinpoppen, H.; Becker, U. VUV and Soft X-Ray Photoionization; Springer US: Boston, 1996.

(3) Yeh, J. J.; Lindau, I. Atomic Subshell Photoionization Cross Sections and Asymmetry Parameters: $1<\mathrm{Z}<103$. At. Data Nucl. Data Tables 1985, 32, 1-155.

(4) Sukhorukov, V. L.; Petrov, I. D.; Lagutin, B. M.; Ehresmann, A.; Schartner, K.-H.; Schmoranzer, H. Many-Electron Dynamics of Atomic Processes Studied by PhotonInduced Fluorescence Spectroscopy. Phys. Rep. 2018, 786, 1-60.

(5) Fano, U. Effects of Configuration Interaction on Intensities and Phase Shifts. Phys. Rev. 1961, 124, 1866-1878.

(6) Codling, K.; Madden, R. P.; Ederer, D. L. Resonances in the Photo-Ionization Continuum of Ne I (20-150 eV). Phys. Rev. 1967, 155, 26-37. 
(7) Hergenhahn, U. Interatomic and Intermolecular Coulombic Decay: The Early Years. J. Electron Spectrosc. Relat. Phenom. 2011, 184, 78-90.

(8) Jahnke, T. Interatomic and Intermolecular Coulombic Decay: The Coming of Age Story. J. Phys. B: At., Mol. Opt. Phys. 2015, 48, 082001.

(9) Cederbaum, L. S.; Zobeley, J.; Tarantelli, F. Giant Intermolecular Decay and Fragmentation of Clusters. Phys. Rev. Lett. 1997, 79, 4778-4781.

(10) Kamke, W.; Kamke, B.; Kiefl, H. U.; Hertel, I. V. Intramolecular Penning Ionization in Benzonitrile-Rare Gas Clusters. Chem. Phys. Lett. 1985, 122, 356-360.

(11) Golan, A.; Ahmed, M. Ionization of Water Clusters Mediated by Exciton Energy Transfer from Argon Clusters. J. Phys. Chem. Lett. 2012, 3, 458-462.

(12) Buchta, D.; Krishnan, S. R.; Brauer, N. B.; Drabbels, M.; O’Keeffe, P.; Devetta, M.; Di Fraia, M.; Callegari, C.; Richter, R.; Coreno, M. et al. Charge Transfer and Penning Ionization of Dopants in or on Helium Nanodroplets Exposed to EUV Radiation. J. Phys. Chem. A 2013, 117, 4394-4403.

(13) Trinter, F.; Williams, J. B.; Weller, M.; Waitz, M.; Pitzer, M.; Voigtsberger, J.; Schober, C.; Kastirke, G.; Müller, C.; Goihl, C. et al. Vibrationally Resolved Decay Width of Interatomic Coulombic Decay in HeNe. Phys. Rev. Lett. 2013, 111, 233004.

(14) Mhamdi, A.; Trinter, F.; Rauch, C.; Weller, M.; Rist, J.; Waitz, M.; Siebert, J.; Metz, D.; Janke, C.; Kastirke, G. et al. Resonant Interatomic Coulombic Decay in HeNe: Electron Angular Emission Distributions. Phys. Rev. A 2018, 97, 053407.

(15) Najjari, B.; Voitkiv, A. B.; Müller, C. Two-Center Resonant Photoionization. Phys. Rev. Lett. 2010, 105, 153002.

(16) Gokhberg, K.; Trofimov, A. B.; Sommerfeld, T.; Cederbaum, L. S. Ionization of Metal 
Atoms Following Valence-Excitation of Neighbouring Molecules. Europhys. Lett. 2005, 72, 228-234.

(17) Siska, P. E. Molecular-Beam Studies of Penning Ionization. Rev. Mod. Phys. 1993, 65, $337-412$.

(18) Björneholm, O.; Federmann, F.; Joppien, M.; Fössing, F.; Kakar, S.; von Pietrowski, R.; Möller, T. Valence- and Inner-Shell Spectroscopy on Rare-Gas Clusters. Surf. Rev. Lett. 1996, 3, 299-306.

(19) Fasshauer, E.; Förstel, M.; Pallmann, S.; Pernpointner, M.; Hergenhahn, U. Using ICD for Structural Analysis of Clusters: A Case Study on NeAr Clusters. New J. Phys. 2014, 16, 103026.

(20) Lundwall, M.; Pokapanich, W.; Bergersen, H.; Lindblad, A.; Rander, T.; Ohrwall, G.; Tchaplyguine, M.; Barth, S.; Hergenhahn, U.; Svensson, S. et al. Self-Assembled Heterogeneous Argon/Neon Core-Shell Clusters Studied by Photoelectron Spectroscopy. J. Chem. Phys. 2007, 126, 214706.

(21) Förstel, M.; Mucke, M.; Arion, T.; Lischke, T.; Barth, S.; Ulrich, V.; Öhrwall, G.; Björneholm, O.; Hergenhahn, U.; Bradshaw, A. M. Observation of Electronic Energy Bands in Argon Clusters. Phys. Rev. B 2010, 82, 125450.

(22) Gibson, N. D.; Risley, J. S. Absolute Measurements of Optical Oscillator Strengths of Noble-Gas Resonance Lines. Phys. Rev. A 1995, 52, 4451-4456.

(23) Fasshauer, E. Non-Nearest Neighbour ICD in Clusters. New J. Phys. 2016, 18, 043028.

(24) Förstel, M.; Mucke, M.; Arion, T.; Lischke, T.; Pernpointner, M.; Hergenhahn, U.; Fasshauer, E. Long-Range Interatomic Coulombic Decay in ArXe Clusters: Experiment and Theory. J. Phys. Chem. C 2016, 120, 22957-22971. 
(25) Mucke, M.; Förstel, M.; Lischke, T.; Arion, T.; Bradshaw, A. M.; Hergenhahn, U. Performance of a Short "Magnetic Bottle" Electron Spectrometer. Rev. Sci. Instrum. 2012, 83, 063106.

(26) Hans, A.; Schmidt, P.; Ozga, C.; Hartmann, G.; Holzapfel, X.; Ehresmann, A.; Knie, A. Extreme Ultraviolet to Visible Dispersed Single Photon Detection for Highly Sensitive Sensing of Fundamental Processes in Diverse Samples. Materials 2018, 11, 869. 